\title{
OPEN Phylogenetic analysis of cell-cycle regulatory proteins within the Symbiodiniaceae
}

\author{
Lucy M. Gorman ${ }^{1}$, Shaun P. Wilkinson ${ }^{1}$, Sheila A. Kitchen ${ }^{2}$, Clinton A. Oakley ${ }^{1}$, \\ Arthur R. Grossman ${ }^{3}$, Virginia M. Weis ${ }^{4}$ \& Simon K. Davy ${ }^{1 凶}$
}

In oligotrophic waters, cnidarian hosts rely on symbiosis with their photosynthetic dinoflagellate partners (family Symbiodiniaceae) to obtain the nutrients they need to grow, reproduce and survive. For this symbiosis to persist, the host must regulate the growth and proliferation of its symbionts. One of the proposed regulatory mechanisms is arrest of the symbiont cell cycle in the $G_{1}$ phase, though the cellular mechanisms involved remain unknown. Cell-cycle progression in eukaryotes is controlled by the conserved family of cyclin-dependent kinases (CDKs) and their partner cyclins. We identified CDKs and cyclins in different Symbiodiniaceae species and examined their relationship to homologs in other eukaryotes. Cyclin proteins related to eumetazoan cell-cycle-related cyclins A, B, D, G/I and $Y$, and transcriptional cyclin $L$, were identified in the Symbiodiniaceae, alongside several alveolatespecific cyclin A/B proteins, and proteins related to protist P/U-type cyclins and apicomplexan cyclins. The largest expansion of Symbiodiniaceae cyclins was in the P/U-type cyclin groups. Proteins related to eumetazoan cell-cycle-related CDKs (CDK1) were identified as well as transcription-related CDKs. The largest expansion of CDK groups was, however, in alveolate-specific groups which comprised 11 distinct CDK groups (CDKA-J) with CDKB being the most widely distributed CDK protein. As a result of its phylogenetic position, conservation across Symbiodiniaceae species, and the presence of the canonical CDK motif, CDKB emerged as a likely candidate for a Saccharomyces cerevisiae Cdc28/ Pho85-like homolog in Symbiodiniaceae. Similar to cyclins, two CDK-groups found in Symbiodiniaceae species were solely associated with apicomplexan taxa. A comparison of Breviolum minutum CDK and cyclin gene expression between free-living and symbiotic states showed that several alveolate-specific CDKs and two P/U-type cyclins exhibited altered expression in hospite, suggesting that symbiosis influences the cell cycle of symbionts on a molecular level. These results highlight the divergence of Symbiodiniaceae cell-cycle proteins across species. These results have important implications for host control of the symbiont cell cycle in novel cnidarian-dinoflagellate symbioses.

Many cnidarians in the marine environment, including reef-building corals, form symbiotic relationships with photosynthetic dinoflagellates from the family Symbiodiniaceae ${ }^{1}$. These dinoflagellate symbionts are located in host gastrodermal cells inside symbiosomes (vacuoles consisting of a host-derived membrane) ${ }^{2}$. This closely integrated intracellular relationship indicates that symbiont population maintenance by the host was likely integral to the evolution of the symbiosis ${ }^{1,3}$. To date, most studies examining symbiont cell division in hospite have focused on nutrient availability ${ }^{4-9}$. However, symbiont growth rate appears to be controlled by more than nutrient limitation, as nutrient-replete symbionts in hospite still have a growth rate that is less than $20 \%$ of symbionts ex hospite $e^{5}$.

Besides nutrient control, other proposed host regulatory mechanisms of symbiont growth and proliferation include pre-mitotic cell-cycle control and post-mitotic autophagy, expulsion and apoptosis ${ }^{1,7,10,11}$. However, the contribution of each mechanism towards the regulation of symbiont biomass, from the onset to the stabilisation of the symbiosis, is unknown. Smith and Muscatine 7 proposed that the main control of a dampened symbiont growth rate in hospite is from the cnidarian host arresting the cell cycle of its resident symbionts. In the eukaryotic cell cycle there is one quiescent stage $\left(G_{0}\right)$ and four subsequent cycling phases: $G_{1}$ (gap 1) where cells grow and are sensitive to extracellular cues such as growth factors ${ }^{12}$; $S$ (synthesis) where genomic DNA is replicated and synthesised ${ }^{13} ; G_{2}$ (gap 2), where DNA breaks that occur during the $S$ phase are repaired before mitosis ${ }^{14}$;

\footnotetext{
${ }^{1}$ School of Biological Sciences, Victoria University of Wellington, Wellington 6140, New Zealand. ${ }^{2}$ Division of Biology and Biological Engineering, California Institute of Technology, Pasadena, CA 91125, USA. ${ }^{3}$ Department of Plant Biology, The Carnegie Institution for Science, Stanford, CA 94305, USA. 'Department of Integrative Biology, Oregon State University, Corvallis, OR 97331, USA. ${ }^{\boxplus e m a i l: ~ S i m o n . D a v y @ v u w . a c . n z ~}$
} 


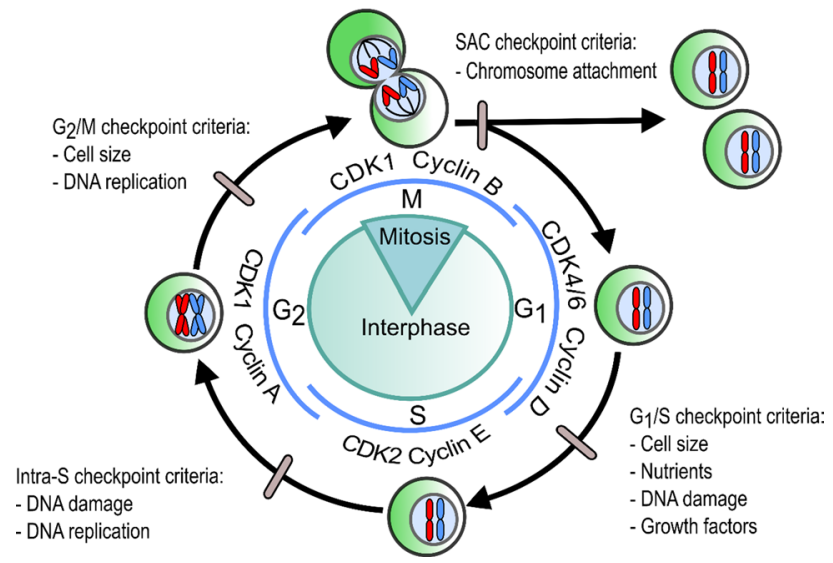

Figure 1. The generalised cell cycle in eumetazoans illustrating the cell-cycle phase and regulatory checkpoints (grey ovals) with their complementary criteria that must be met for the cell cycle to progress through that checkpoint. Blue lines depict the formation of cell-cycle phase associated CDK-cyclin complexes in Homo sapiens. SAC Spindle Assembly Checkpoint.

and $\mathrm{M}$ (mitosis), where two equal copies of the chromosomes are distributed between the two cells ${ }^{13}$. In the sea anemone Exaiptasia pallida ('Aiptasia'), $80 \%$ of the resident symbionts were shown to be arrested at the $G_{0} / G_{1}$ phase compared with $40-55 \%$ in culture ${ }^{7}$.

Once a cell enters the cell cycle, it can be arrested at a series of cell-cycle checkpoints (Fig. 1). These checkpoints monitor the integrity and correct progression of the cell cycle with each checkpoint containing criteria that must be met for a cell to progress to the next stage of the cycle ${ }^{15,16}$. Each checkpoint is regulated by cyclindependent kinases (CDKs) and their partner cyclins ${ }^{17}$. Once a cell meets its checkpoint criteria, cyclins are synthesised and bind to their partner $\mathrm{CDKs}^{17}$. Cyclins regulate the catalytic activity of CDKs ${ }^{18}$. These CDK-cyclin complexes can directly trigger cell-cycle progression (Fig. 1) or indirectly trigger cell-cycle progression through a variety of other downstream events such as transcription, DNA damage repair, proteolytic degradation and metabolism ${ }^{19}$. Table S1 summarises the cell-cycle stage and roles of individual CDK and cyclin proteins. CDKcyclin complexes in Homo sapiens are shown in Fig. 1; however, the type and quantity of CDKs and cyclins are specific to a particular species ${ }^{17}$.

Identification of cell-cycle proteins in the Symbiodiniaceae is just beginning, with a study by Cato et al. ${ }^{20}$ finding 10 distinct CDKs and 15 distinct cyclin genes in the genome of Breviolum minutum. In the same study ${ }^{20}$, qPCR analysis revealed that a cyclin B2/CDK1 pair was expressed during the $\mathrm{G}_{1} / \mathrm{S}$ phase transition in cultured B. minutum. As there are at least nine genera of Symbiodiniaceae ${ }^{21,22}$, determining whether cell-cycle proteins present in B. minutum are conserved across the Symbiodiniaceae will inform our understanding of cell-cycle progression and cellular growth rates in this family. For example, a recent study ${ }^{23}$ comparing cell-cycle progression between four Symbiodiniaceae genera (Symbiodinium, Breviolum, Cladocopium and Durusdinium) in culture, found that the proportion of the population progressing through the cell cycle was different between genera, resulting in differing growth rates. Similarly, different Symbiodiniaceae species have been shown to have different proliferation rates and reach different densities within the same host ${ }^{24-27}$, with inherent differences in cell-cycle machinery between species being one possible explanation. The current study represents the first attempt to identify and describe cell-cycle proteins across diverse Symbiodiniaceae species and provides a basis for future research.

\section{Materials and methods}

Identification of Symbiodiniaceae CDKs and cyclins. Twenty-seven Symbiodiniaceae transcriptome and genome databases were acquired from publicly available sources (Table S2). Cyclins and CDKs from two free-living Symbiodiniaceae species, Effrenium voratum ${ }^{28}$ and Fugacium kawagutii ${ }^{29}$, were compared with symbiotic species (Symbiodinium microadriaticum, S. tridacnidorum, Breviolum minutum, B. aenigmaticum, B. pseudominutum, B. psygmophilum, Cladocopium goreaui, Cladocopium genotypes C15 and C92 and Durusdinium trenchii). Profile hidden Markov models (pHMMs) were obtained from the PFAM 31.0 database for the cyclin N terminal domain (PF00134) and CDK conserved domain (PF00069). The pHMM models were re-trained using CDKs and cyclins from eukaryotic organisms closely related to Symbiodiniaceae (e.g. Apicomplexa) using the aphid R package ${ }^{30}$.

The Symbiodiniaceae databases were then queried with the updated pHMM models using an optimal alignment homology search to find putative cyclin and CDK sequences (Fig. S1). Sequences with log-odds similarity scores $>50$ were retained for cyclins and CDKs. The cyclin model returned 119 sequences and the CDK model returned 6032 sequences. Due to the high abundance of Symbiodiniaceae CDK sequences returned from the model, the collected CDK sequences from the pHMM model were examined further using conserved CDK motifs (Table S3) ${ }^{31-34}$. If the CDK contained a motif that when BLASTp searched against the NCBI non-redundant database matched to a CDK, the sequence was retained for further analysis. All 119 cyclins retrieved by the model were also searched, and were included in the analysis if the highest-scoring sequence was annotated as a cyclin 
or CDK and had an E value $\leq 1 \times 10^{-5}$. Owing to the lack of information available for CDKs and cyclins in other unicellular marine eukaryotes, several taxa (Table S5) were chosen for screening through the trained pHMM models to identify putative cyclin and CDK sequences, allowing possible alveolate-specific groups to be identified.

Sequence alignment and phylogenetic analysis. Phylogenetic trees were generated twice. The sequence alignment for the first set of trees was aligned to just the conserved cyclin N (PFAM ID:PF00134) and protein kinase domains (PFAM ID: PF00069), which were used to determine distinct phylogenetic groups of Symbiodiniaceae cyclins and CDKs. These were later used to identify other similar sequences from the Symbiodiniaceae databases.

The first trees were generated by aligning the putative CDKs and cyclins in the aphid R package ${ }^{30}$ (along with other eukaryotic cyclins and CDKs) and the best substitution model was selected by ProTest (v3.4) ${ }^{35}$. Both alignments had an appropriate evolutionary model of PROTOGAMMAAUTO, which was then used to infer maximum-likelihood trees in RAxML (v8.2.12) ${ }^{36}$. Bootstrap support was used to find the distinct phylogenetic groupings among Symbiodiniaceae CDKs and cyclins $(n=1000)$ by using the topology of the tree with the highest log-likelihood score. Trees were rooted using the H. sapiens MAPK (NP_002737.2) gene for the CDK tree and H. sapiens CABLES1 (NP_112492.2) and H. sapiens CABLES2 (NP_001094089.1) for the cyclin tree based on a previous study on animal cyclins and $\mathrm{CDKs}^{37}$. Symbiodiniaceae candidate proteins from distinct phylogenetic $\mathrm{CDK}$ and cyclin groups were used to perform custom BLASTp searches (Table S4) in Geneious v.11.1.5 (Biomatters Ltd.) against the 27 Symbiodiniaceae databases used in this study, to ensure that all putative CDKs and cyclins were identified. The first 10 Symbiodiniaceae proteins with the highest E-value $\left(\leq 1 \times 10^{-5}\right)$ that were not previously identified by the pHMM model, and that identified a CDK or cyclin on the NCBI nr database in BLASTp searches, were collected from each Symbiodiniaceae database for each of the candidate proteins. These newly identified Symbiodiniaceae sequences were added to the previously collected sequences through the pHMM models and together these were entered into CD-Hit $\mathrm{v} 4.8^{38}$ to remove isoforms and redundant proteins using a similarity threshold of $90 \%$.

Once redundant proteins and isoforms were removed, Symbiodiniaceae sequences were submitted to InterProScan ${ }^{39}$ to identify CDK and cyclin domains. Due to the low-quality annotations in Symbiodiniaceae databases $^{40}$, many sequences contained regions that coded other proteins, therefore the alignments were trimmed manually in Geneious v.11.1.5 to CDK- (PFAM ID: PF00069; PANTHER ID: PTHR24056) and cyclin-(PFAM ID:PF00134, PF02984, PF16899 and PF08613; PANTHER ID: PTHR10177) annotated domains. The final CDK alignment for the second phylogenetic analysis was 465 amino acids (aa) long, and contained 177 Symbiodiniaceae sequences and $50 \mathrm{CDKs}$ from other eukaryotes (Supplementary File S1), whereas the cyclin alignment was 395 aa long and contained 191 Symbiodiniaceae sequences and 54 cyclins from other eukaryotes (Supplementary File S2). All CDK and cyclin families from Homo sapiens were included in the trees to create the correct topologies, and CDKs and cyclins from other model organisms, including Saccharomyces cerevisiae and Arabidopsis thaliana, were only included if Symbiodiniaceae proteins were related to them.

Final CDK and cyclin alignments were run through ProTest (v3.4) $)^{35}$ as described previously. Maximumlikelihood trees were then run in PhyML (v3.1) $)^{41}$ using the Akaike information criterion, which corresponded to the $\mathrm{LG}+\mathrm{I}+\mathrm{G}+\mathrm{F}$ model for the CDK alignment with a proportion of invariable sites of 0.039 and a gamma shape parameter of 1.195 , and the $L G+G+F$ model for cyclin alignments with a gamma shape parameter of 2.331. Due to the quantity of sequences in the tree, an approximate likelihood ratio test (aLRT) was used for branch support instead of bootstrap support ${ }^{42}$, however it has been shown to be very similar in calculating correct branch supports ${ }^{43}$. Based on a comparison of correct branch topologies determined by bootstrap support and SH-values ${ }^{43}$, true Symbiodiniaceae CDK and cyclin homologs were determined by branches containing an $\mathrm{SH}$-value $>0.8$. Trees were rooted as described previously. Trees were edited in the Interactive Tree of Life (iToL) software v.5.6.3 ${ }^{44}$. The nomenclature of protein groups that did not phylogenetically group with other well-classified CDKs or cyclins was attributed by using BLAST searches against the NCBI nr database.

Cyclin and CDK gene expression of Breviolum minutum. To explore expression of cyclins and CDKs in Symbiodiniaceae, RNA-Seq reads were analysed from a recent study by Maor-Landaw et al. ${ }^{45}$ on the expression of cultured $(n=3)$ and freshly isolated Breviolum minutum $(n=3)$ from the sea anemone Exaiptasia diaphana (=pallida) (SRA PRJNA544863). Reads were aligned to the $B$. minutum genome assembly ${ }^{46}$ using STAR v2.7.1a in two-pass mode ${ }^{47}$ and read counts were extracted from the alignments with featureCounts v1.6.3 ${ }^{48}$. Differential expression analysis was completed using the exact test in $\mathrm{EdgeR}^{49}$ on TMM normalized counts of the cultured and isolated B. minutum. Differentially expressed genes (DEGs) were those with Benjamini-Hochberg adjusted $p$-values $<0.05$. Cyclins and CDKs identified in B. minutum were selected from the list of DEGs to generate a heat map in the $\mathrm{R}$ environment ${ }^{50}$, using the mean-variance modelling at the observational level (voom) ${ }^{51}$ of $\log _{2}$-transformed counts per million (CPM).

\section{Results and discussion}

Characterisation and phylogenetic positioning of Symbiodiniaceae CDK sequences. Eukaryotic organisms contain different numbers of CDK proteins, ranging from three in premetazoans, to 20 in eumetazoans such as Homo sapiens ${ }^{37}$. A total of 177 unique Symbiodiniaceae CDK gene copies were identified across six genera (Table 1). CDK gene copy numbers were the highest in Cladocopium goreaui, which contained 16 CDK copies. Interestingly, no CDKs related to the CDK4/6 family nor their cyclin partners (cyclin E) were found in Symbiodiniaceae using the databases referenced in this study (Table 1; Fig. 2). This agrees with findings for plants and many protists, in which there is also an absence of the CDK4/6 family and cyclin E in most pre-metazoan lineages ${ }^{37}$. 


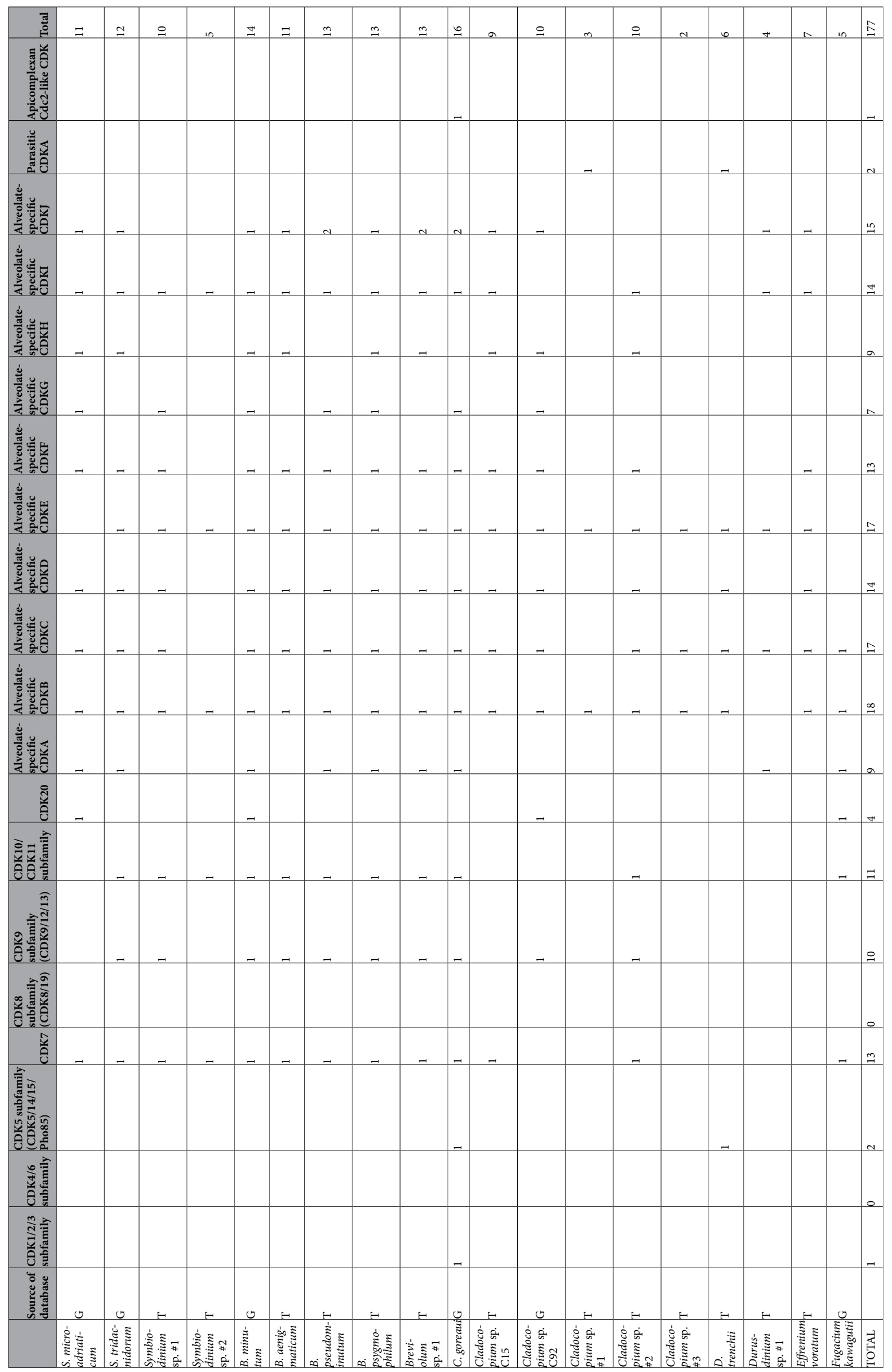




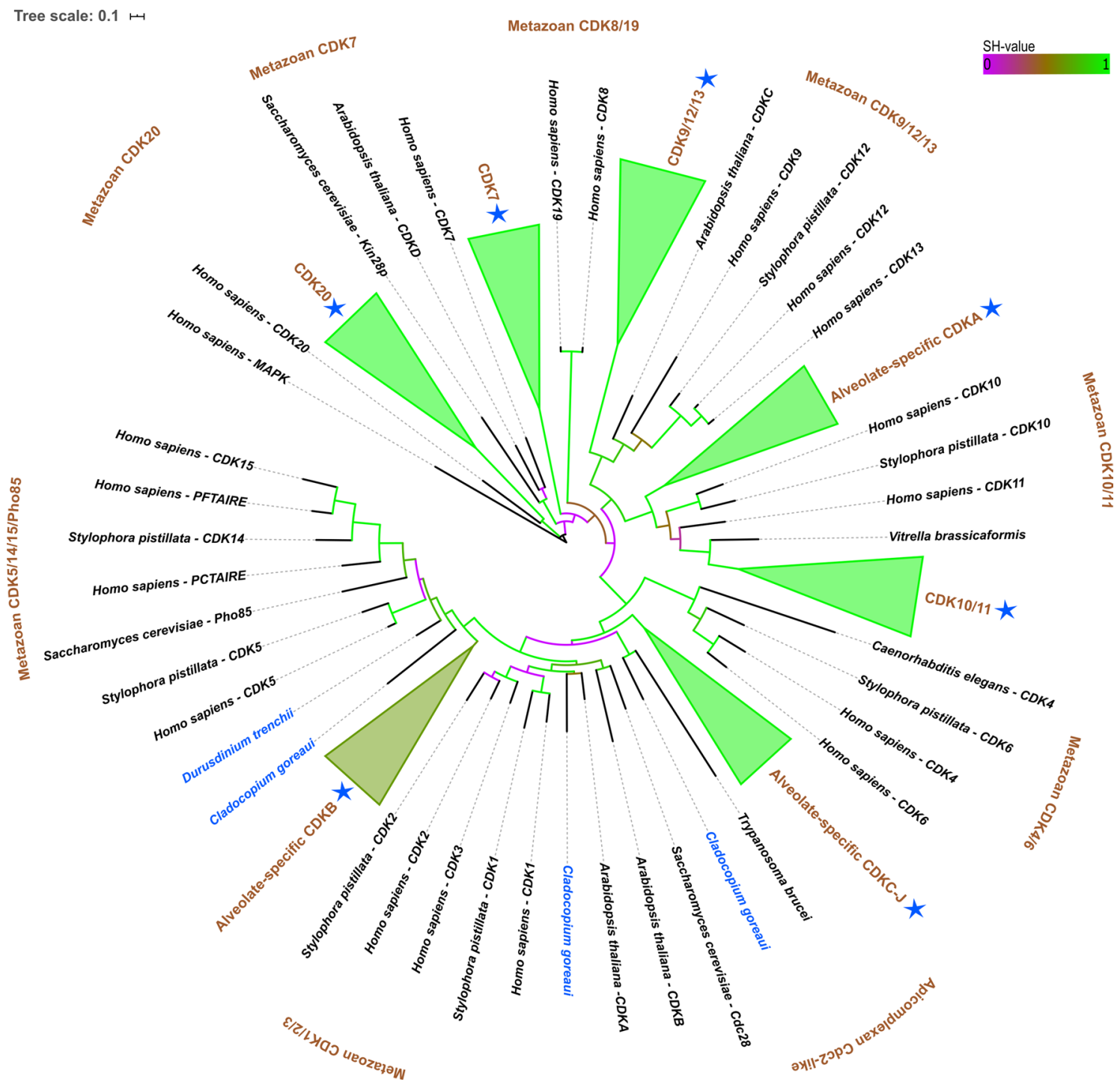

Figure 2. Collapsed phylogenetic tree of CDKs in the Symbiodiniaceae. Colour of branches corresponds to aLRT support (SH-value). Purple branches correspond to $\mathrm{SH}$-values below 0.5 , brown branches correspond to $\mathrm{SH}$-values near 0.5 , and green branches correspond to $\mathrm{SH}$-values close to 1 . Symbiodiniaceae species are written in blue, and blue stars depict collapsed branches containing Symbiodiniaceae species. The tree was made using PhyML(v3.1) ${ }^{41}$ and visualised using the Interactive Tree of Life software (v5.6.3) ${ }^{44}$.

Some of the Symbiodiniaceae CDKs showed high sequence similarity to eumetazoan CDKs, however the largest expansion of CDKs was within the alveolate-specific CDK groups (Table 1, Fig. 2). A previous study ${ }^{20}$ investigating Symbiodiniaceae cell-cycle proteins found four B. minutum-specific CDKs. Here we show that three of those four CDKs are also present across other Symbiodiniaceae species (alveolate-specific CDKG/H/J-Table 1; Supplementary Fig. S2). In the previous study ${ }^{20}$, the B. minutum CDKs (alveolate-specific CDKG/H/J) did not change their expression with cell-cycle phase when in a free-living state. However, our analysis of the previously published RNA-Seq data ${ }^{45}$ shows that symbiosis alters the expression of B. minutum CDKG and CDKH, which were both up-regulated in hospite compared to when in culture (Table S6; Fig. 3).

The most common CDK identified in Symbiodiniaceae was an alveolate-specific CDK (CDKB) with gene copies found across 18 species in the five Symbiodiniaceae genera examined (Table 1). Symbiodiniaceae proteins in the CDKB group contained the canonical CDK motif PSTAIRE (Table 2). The CDKB sister clade is the Pho85/ CDK5 subfamily ( $\mathrm{SH}$-value 0.95 ), which is sister to the metazoan CDK1/S. cerevisae Cdc28, with strong branch support (SH-value = 1; Supplementary Fig. S2). CDK1/Cdc28 is the primary cell-cycle regulator from yeast to humans $^{52-54}$, however Pho85 has been shown to have overlapping roles with Cdc28, phosphorylating many of the same substrates ${ }^{55}$. The primary roles of Pho85 include responding to environmental cues via the induction of signals that inform the cell whether conditions are adequate for cell division and nutrient metabolism ${ }^{56}$. As Symbiodiniaceae proliferate in response to increased nutrients ${ }^{5}$, they may have evolved CDKs that possess similar functions for linking external stimuli (e.g. environmental nitrogen and phosphorus levels) to cell-cycle progression. Furthermore, our analysis of the RNA-Seq data comparing cultured versus freshly-isolated B. minutum ${ }^{45}$ 


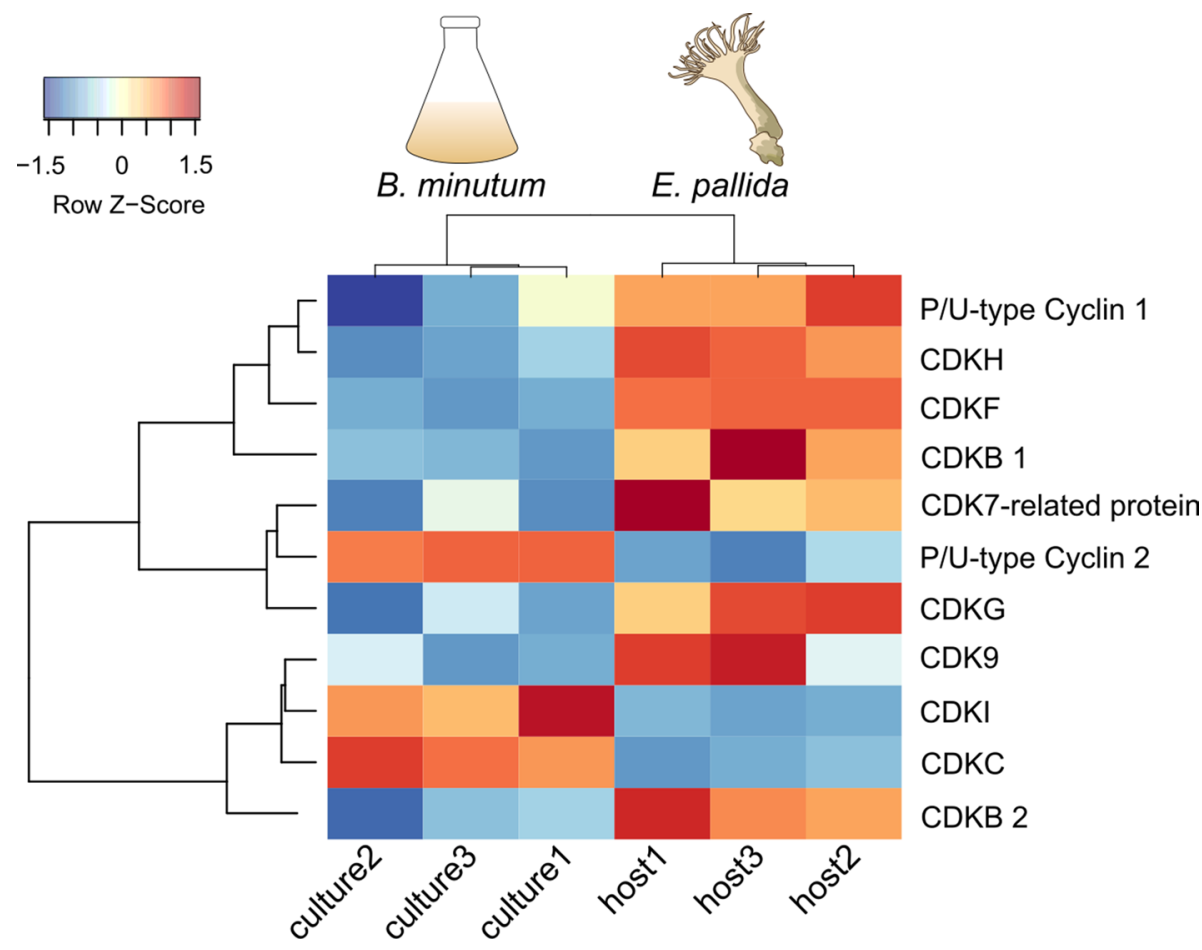

Figure 3. Heat map comparison of Breviolum minutum cyclin and CDK gene expression between culture and cells freshly isolated from Exaiptasia pallida. Red corresponds to a higher Z-score and gene up-regulation whilst blue corresponds to a lower Z-score and down-regulation.

\begin{tabular}{|l|l|}
\hline CDK group & Symbiodiniaceae motif \\
\hline CDK1/2/3 subfamily & PSTALRE \\
\hline CDK4/6 subfamily & N/A \\
\hline CDK5 subfamily (CDK5/14/15/Pho85) & PCTAIRE \\
\hline CDK7 & (G/S)TALRE \\
\hline CDK8 subfamily (CDK8/19) & N/A \\
\hline CDK9/12/13 & $\mathrm{P}(\mathrm{A} / \mathrm{T} / \mathrm{S}) \mathrm{T}(\mathrm{S} / \mathrm{A} / \mathrm{C})(\mathrm{I} / \mathrm{V}) \mathrm{RE}$ \\
\hline CDK10/11 & $\mathrm{P}(\mathrm{V} / \mathrm{S})(\mathrm{P} / \mathrm{A} / \mathrm{S}) \mathrm{S}(\mathrm{L} / \mathrm{I}) \mathrm{RE}$ \\
\hline CDK20 & $\mathrm{PWFSAERE}$ \\
\hline Alveolate-specific CDKA & $\mathrm{P}(\mathrm{K} / \mathrm{R})(\mathrm{I} / \mathrm{S}) \mathrm{SLRE}$ \\
\hline Alveolate-specific CDKB & $\mathrm{PSTAIRE}$ \\
\hline Alveolate-specific CDKC & $\mathrm{PSTAIRE}$ \\
\hline Alveolate-specific CDKD & $\mathrm{PSTALRE/EHQLRRE}$ \\
\hline Alveolate-specific CDKE & $\mathrm{P}(\mathrm{G} / \mathrm{S}) \mathrm{TA}(\mathrm{I} / \mathrm{L}) \mathrm{RE}$ \\
\hline Alveolate-specific CDKF & $(\mathrm{S} / \mathrm{P})(\mathrm{A} / \mathrm{P})(\mathrm{T} / \mathrm{H} / \mathrm{Y} / \mathrm{Q})(\mathrm{T} / \mathrm{A} / \mathrm{V})(\mathrm{I} / \mathrm{L}) \mathrm{RE}$ \\
\hline Alveolate-specific CDKG & $\mathrm{S}(\mathrm{A} / \mathrm{T}) \mathrm{Q}(\mathrm{V} / \mathrm{A}) \mathrm{LRE}$ \\
\hline Alveolate-specific CDKH & $(\mathrm{S} / \mathrm{T}) \mathrm{S}(\mathrm{Y} / \mathrm{F})(\mathrm{S} / \mathrm{A})(\mathrm{L} / \mathrm{I}) \mathrm{RE}$ \\
\hline Alveolate-specific CDKI & $\mathrm{P}(\mathrm{T} / \mathrm{A})(\mathrm{T} / \mathrm{A})(\mathrm{S} / \mathrm{T} / \mathrm{A})(\mathrm{I} / \mathrm{L}) \mathrm{RE}$ \\
\hline Alveolate-specific CDKJ & $\mathrm{P}(\mathrm{T} / \mathrm{A}) \mathrm{TALRE}$ PAVA(L/M)RE \\
\hline Parasite CDKA & $\mathrm{PSTAIRE}$ \\
\hline Apicomplexan Cdc2-like CDK & $\mathrm{PQTALRE}$ \\
\hline
\end{tabular}

Table 2. Conserved motifs found in the Symbiodiniaceae CDK genes.

suggests that two CDKB genes are up-regulated in symbiosis (Table S6; Fig. 3). We hypothesise that, due to its phylogenetic grouping, conserved motif, widespread presence across Symbiodiniaceae and up-regulation in the symbiotic state, CDKB may be a homolog of Cdc28/Pho85 and a primary cell-cycle regulator in Symbiodiniaceae. This hypothesis requires confirmation. 


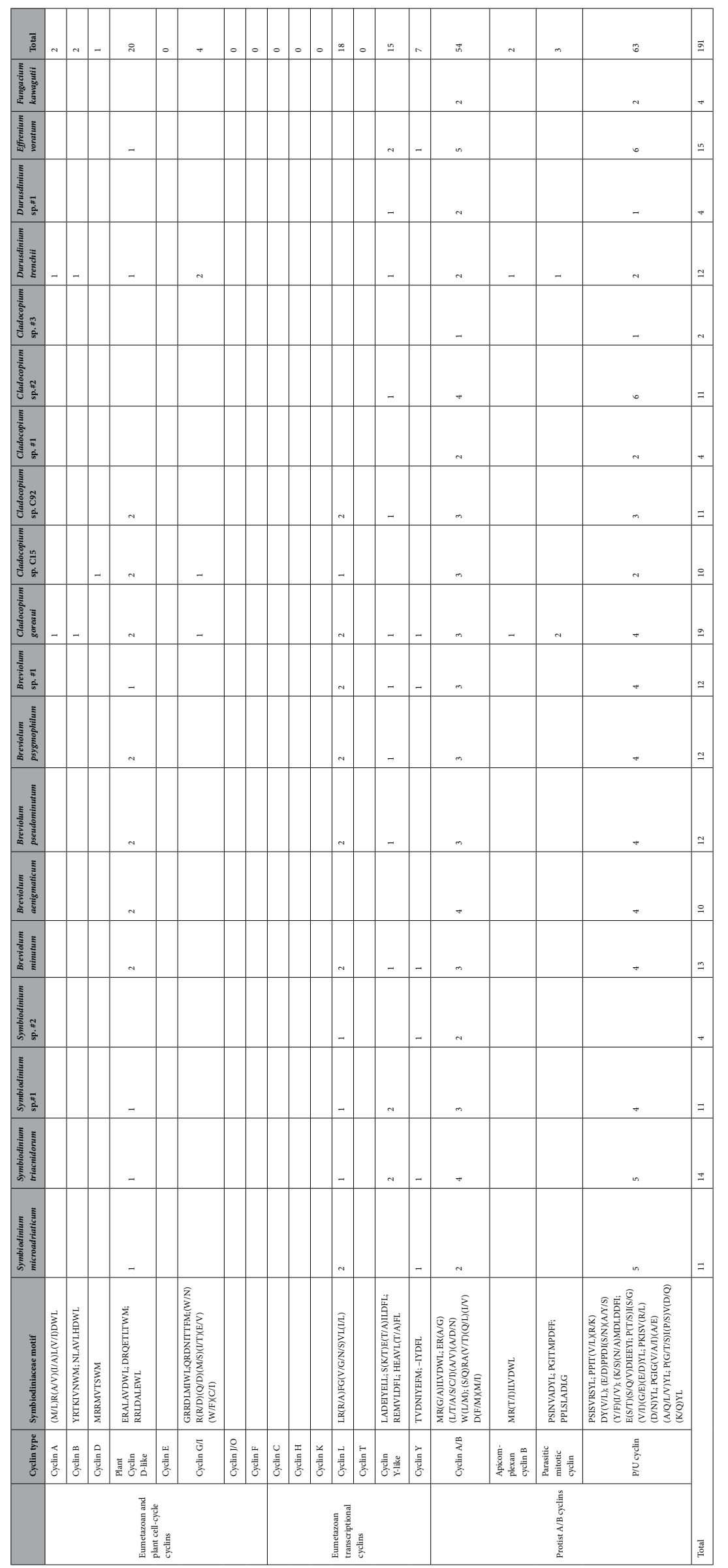

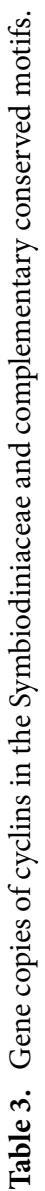




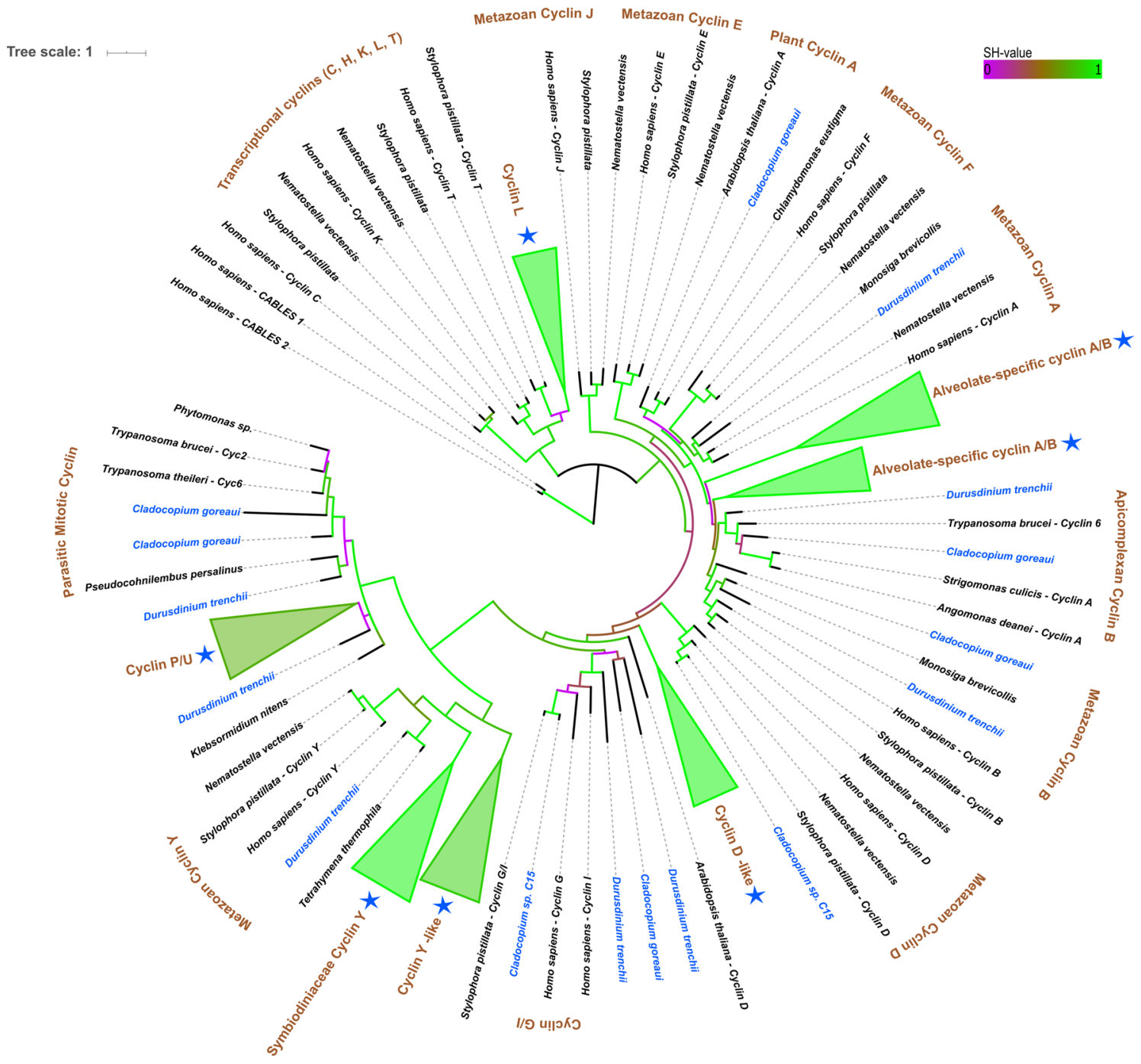

Figure 4. Collapsed phylogenetic tree of cyclins in the Symbiodiniaceae. Colour of branches corresponds to aLRT support (SH-value). Purple branches correspond to SH-values below 0.5, brown branches correspond to $\mathrm{SH}$-values near 0.5 , and green branches correspond to $\mathrm{SH}$-values close to 1 . Symbiodiniaceae species are written in blue, and blue stars depict collapsed branches containing Symbiodiniaceae species. The tree was made using PhyML(v3.1) ${ }^{41}$ and visualised using the Interactive Tree of Life software (v.5.6.3) ${ }^{44}$.

Proteins related to eumetazoan transcriptional CDK subfamilies (CDK9/12/13 (SH-value $=0.89)$, CDK10/11 $(\mathrm{SH}$-value $=0.89)$ and CDK20 $(\mathrm{SH}$-value $=0.93))$ were also present in Symbiodiniaceae (Table 1; Supplementary Fig. S2). Amongst transcriptional roles, the CDK10/11 subfamily has also been proposed to have roles in cellcycle progression during the $\mathrm{G}_{2} / \mathrm{M}$ phase (Table $\left.\mathrm{S} 1\right)^{57}$. However, in B. minutum, CDK20, CDK9 and CDK11 expression did not change with cell-cycle phase ${ }^{20}$, highlighting their similarity to metazoan CDK20, CDK9 and CDK11, which are predominantly transcriptional CDKs and indirectly related to the cell cycle ${ }^{58}$. Previous studies ${ }^{20}$ have reported an absence of CDK7 in B. minutum, however this study found a CDK7-related gene (confirmed via BLAST searches on the NCBI nr database) across 13 different Symbiodiniaceae species (Supplementary Fig. S2). The difference in results may be explained, in part, by the Symbiodiniaceae CDK7 being phylogenetically distant from the metazoan CDK7 and yeast CDK7 homolog (Kin28p), grouping separately and with no concrete relationship to any other CDK included in this study, possibly owing to its divergence. CDK7 has been discovered in other basal organisms, such as the amoebozoan Dictyostelium purpureum ${ }^{59}$. In metazoans, CDK7 forms part of the cyclin kinase-activating $(\mathrm{CAK})$ complex that activates other CDKs by phosphorylating their T-loop ${ }^{60}$, and inhibition of CDK7 led to the arrest of the cell cycle in proliferating cells ${ }^{61}$. The previously published RNA-Seq data ${ }^{45}$ show that the CDK7-related gene was up-regulated in symbiotic B. minutum (Table S6).

Symbiodinium sp. \#2 contained CDKs and cyclins that are more similar to those of the free-living dinoflagellate Amphidinium (SH-value >0.95) than other Symbiodiniaceae species (Supplementary Fig. S2). CDKs and cyclins that are not present in Amphidinium sp. but are present in Symbiodinium sp. \#2 grouped next to, not with, the other Symbiodiniaceae species ( $\mathrm{SH}$-value $>0.78$ ). This placement may reflect the basal status of Symbiodinium within the Symbiodiniaceae ${ }^{21}$. 


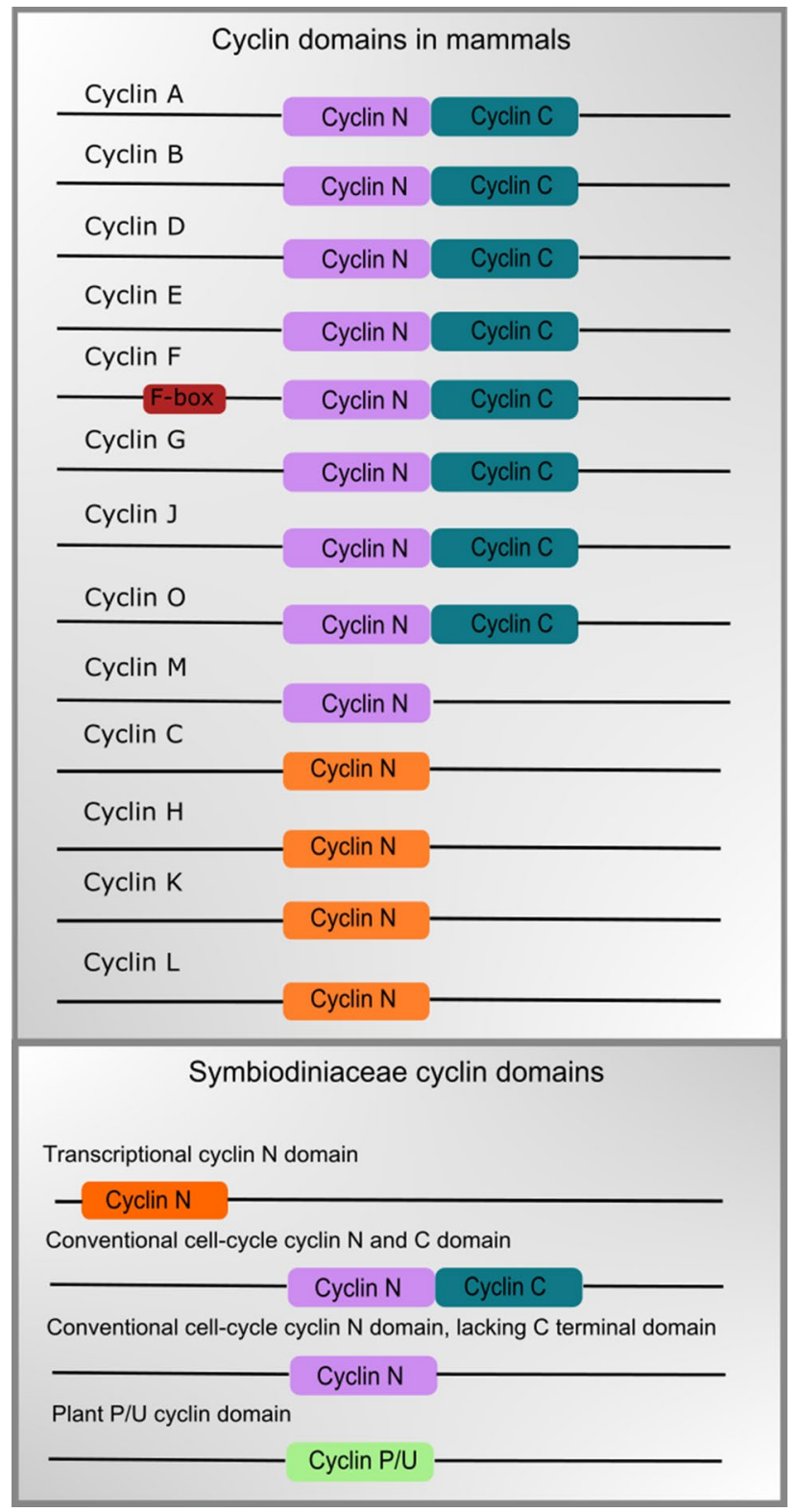

Figure 5. Domain structure of cyclin proteins present in the Symbiodiniaceae.

Several Symbiodiniaceae species contained CDKs found in parasitic taxa. A CDK protein that is related to a gene present in the free-living, facultative pathogenic marine ciliate Pseudocohnilembus persalinus, was found in both D. trenchii and Cladocopium sp. \#1 (SH-value =1), while C. goreaui harbours a CDK related to Cdc2related kinase 6 (CRK6) from Trypanosoma brucei $\left(\mathrm{SH}\right.$-value = 0.97) (Fig. 2, Supplementary Fig. S2). Studies ${ }^{62,63}$ have shown that the loss of T. brucei CRK6 slows cell growth but does not inhibit the cell cycle (contrasting with cell cycle indispensable CRK3 and CRK1), highlighting a function of CRK6 that may not be directly associated with the cell cycle.

Characterisation and phylogenetic positioning of Symbiodiniaceae cyclin sequences. Similar to CDKs, the number of cyclins differs across eukaryotes - from eight in premetazoans to 29 in Homo sapiens ${ }^{37}$. Across the six Symbiodiniaceae genera examined, 191 cyclins were identified (Table 3; Fig. 4). C. goreaui contained the most cyclin gene copies, harbouring 19 distinct copies. Differences in abundance of cell-cycle proteins (cyclins and CDKs) between different Symbiodiniaceae species could be a result of the different database information provided (genomes versus transcriptomes), as if CDKs and cyclins were not expressed at the time of transcriptomic analysis, these may have been missed, thus producing a bias towards genomes harbouring more cyclin and CDK gene copies. Another possible reason for the difference in cyclin and CDK gene copies in the Symbiodiniaceae are gene duplication events, which are followed by genetic drift over time, causing the formation of cell-cycle paralogs with functional divergence in the family. 
All the cyclins found in the Symbiodiniaceae contained one of three distinct domains (Fig. 5): the conventional cell-cycle cyclin $\mathrm{N}$ and $\mathrm{C}$ domains; a cyclin $\mathrm{N}$ domain found nearer the amino terminus than the position of the conventional cell-cycle cyclin $\mathrm{N}$ domain which corresponded phylogenetically to transcriptional cyclins (specifically cyclin L); and a single plant $\mathrm{P} / \mathrm{U}$ cyclin domain that is phylogenetically related to the analogous domain of the Pho80p cyclin in S. cerevisiae.

Proteins related to eukaryotic cell-cycle cyclins A, B, D and G/I, and transcriptional cyclin L were identified in the Symbiodiniaceae, along with proteins related to plant cyclin D, protist/plant $\mathrm{P} / \mathrm{U}$-type cyclin and cyclin Y, as well as genes related to Cyc2 and mitotic Cyc6 from the sister taxon Apicomplexa (Fig. 4; Supplementary Fig. S3). Three phylogenetically distinct groups of cyclins were also present in Symbiodiniaceae, that upon searching the NCBI nr database, matched to alveolate-specific cyclins A/B (Supplementary Fig. S3). Two cyclins previously reported to be $B$. minutum-specific ${ }^{20}$ were found in other Symbiodiniaceae species and belong to the "Plant Cyclin D-like" grouping (Table 3; Supplementary Fig. S3). In metazoans and plants, cyclin D is required for $\mathrm{G}_{1}$ phase progression ${ }^{64}$.

An expansion of the protist/plant P/U-type cyclin groups was found within Symbiodiniaceae, with 63 gene copies being present across six Symbiodiniaceae genera (Table 3, Fig. 4, Supplementary Fig. S3). This finding agrees with the previous study ${ }^{20}$, which found P-type cyclins in B. minutum. Genes within these groups were related to the S. cerevisiae Pho80p cyclin. In S. cerevisiae, the Pho80 subfamily of P/U-type cyclins (Pho80, Pcl6, $\mathrm{Pcl} 7, \mathrm{Pcl} 8$ and $\mathrm{Pcl} 10^{55}$ ) links nutrient availability with cell-cycle progression ${ }^{65}$. In A. thaliana, P/U-type cyclins are implicated in the switch from heterotrophic to autotrophic growth ${ }^{66}$. RNA-Seq data ${ }^{45}$ revealed that two of these P/U type cyclins had contrasting expression (one being up-regulated whilst the other was down-regulated) in hospite versus in culture in B. minutum (Table S6; Fig. 3). Given that nutritional exchange is a fundamental feature of the cnidarian-dinoflagellate symbiosis ${ }^{1}$, and that $\mathrm{P} / \mathrm{U}$ cyclins are involved in glycogen metabolism and carbon source utilisation ${ }^{56,67}$, the differential expression of these cyclins in hospite is unsurprising. Whether the difference in expression is a response to environmental stimuli exclusively experienced in symbiosis, e.g. host-associated factors such as the $\mathrm{pH}$ of the symbiosome in which the alga resides ${ }^{68}$, requires further study. Similar to Symbiodiniaceae, the apicomplexan T. gondii also lacks a cyclin E homolog and instead uses a P-type cyclin for $G_{1}$ phase progression ${ }^{69}$. Symbiodiniaceae may also use P-type cyclins in place of eumetazoan cyclin $\mathrm{E}$, however this requires confirmation.

Twenty two cyclin Y-like gene copies were found across the Symbiodiniaceae. These encompassed two phylogenetic groups, one termed "Cyclin Y" which grouped with eumetazoan Cyclin Y (SH-value = 0.93), and one group of cyclins that grouped as a sister group with the conventional eumetazoan Cyclin Y $(\mathrm{SH}$-value $=0.80)$ that were termed "Cyclin Y-like" (Fig. 4, Supplementary Fig. S3). Cyclin Y is absent in plants and fungi (being replaced by the Pcl class of cyclins in fungi) but is present in animals and protists ${ }^{59}$. In eumetazoans and fungi, cyclin Y and Pcl1 cyclins are the binding partners of CDK14 and Pho85, respectively ${ }^{70,71}$. In yeast, the cyclin Y homolog, Pcl1, is expressed during the $\mathrm{G}_{1}$ phase of the cell cycle ${ }^{70}$ and provides information to the cell, determining whether it passes the START checkpoint, where the yeast cell commits to mitosis ${ }^{56}$. In Drosophila, cyclin Y is required for Wnt signalling by localising the CDK14 kinase to the cell membrane ${ }^{72}$. As Wnt signalling is an indispensable pathway for the long-term viability of cells ${ }^{73}$, the presence of cyclin Y and cyclin-Y like genes in most eukaryotes is predicted.

Uniquely, C. goreaui and D. trenchii both contain cyclins present in two phylogenetic groups that cluster with mitotic cyclins from the dinoflagellate sister taxon, the apicomplexans ${ }^{74}$ (Fig. 4). One group is related to the B-type $\mathrm{G}_{2} / \mathrm{M}$ phase-specific cyclin, Cyc6, in the apicomplexans ( $\mathrm{SH}$-value $>0.98$ ), while the other clusters with Cyc2-like from T. brucei, which is involved in transition from both the $G_{1}$ to $S$ and $G_{2}$ to $M$ phases ${ }^{75}$ (Fig. 4). The correlation in cell-cycle machinery of both cyclins and CDKs between pathogenic protists and D. trenchii, which is reported to colonise hosts during heat stress opportunistically ${ }^{25,76}$ and has a fast growth rate versus other Symbiodiniaceae species in culture ${ }^{23}$, is noteworthy and warrants future investigation.

Cladocopium sp. C15 harbours two cyclins (cyclin D and cyclin G/I) that are related to those in the symbiotic coral, Stylophora pistillata, with strong support ( $\mathrm{SH}$-value $=1$ ). Both Cladocopium sp. C15 cyclin D and G/I share a similar identity (92.1\% and $74.5 \%$, respectively) and similarity (95.7\% and $91.6 \%$, respectively), across the full sequence length to $S$. pistillata cyclins. To account for possible contamination of host material in the Cladocopium sp. C15 transcriptome, the origin of this symbiont was traced ${ }^{77}$. The Cladocopium sp. C15 was found to have been freshly isolated from its host Porites compressa, so host contamination cannot be excluded. This being said, symbiosis has been suggested to drive the formation of paralogous genes involved in host-symbiont interactions due to selective pressure for a more mutualistic partnership between host and symbiont ${ }^{78}$. How the evolution of cell-cycle proteins that share a high similarity between host and symbiont affects biomass co-ordination is deserving of future attention.

\section{Conclusions}

Our study shows the divergence of cell-cycle proteins in the Symbiodiniaceae family and demonstrates that there are several conserved CDK and cyclin groups across the Symbiodiniaceae, though also marked species-specific differences. Which of these conserved cell-cycle proteins are indispensable for cell-cycle progression and which species-specific proteins influence proliferation rates in symbiosis remains unknown. Further study will be required to clarify which CDKs and cyclins are required for Symbiodiniaceae cell-cycle progression, and whether this differs between species and symbiotic states. As annotation of Symbiodiniaceae genomes is challenging ${ }^{79}$, future studies should aim to apply the same comparative analysis across new Symbiodiniaceae genomes to inform cyclin and CDK gene prediction accurately. 
Received: 11 April 2020; Accepted: 28 October 2020

Published online: 24 November 2020

\section{References}

1. Davy, S. K., Allemand, D. \& Weis, V. M. Cell biology of cnidarian-dinoflagellate symbiosis. Microbiol. Mol. Biol. Rev. 76, 229-261 (2012).

2. Wakefield, T. S. \& Kempf, S. C. Development of host-and symbiont-specific monoclonal antibodies and confirmation of the origin of the symbiosome membrane in a cnidarian-dinoflagellate symbiosis. Biol. Bull. 200, 127-143 (2001).

3. Jones, R. J. \& Yellowlees, D. Regulation and control of intracellular algae (= zooxanthellae) in hard corals. Philos. Trans. R. Soc. Lond. B. 352, 457-468 (1997).

4. Hoegh-Guldberg, O. \& Smith, G. J. Influence of the population density of zooxanthellae and supply of ammonium on the biomass and metabolic characteristics of the reef corals Seriatopora hystrix and Stylophora pistillata. Mar. Ecol. Prog. Ser. 57, 173-186 (1989).

5. Hoegh-Guldberg, O. The population dynamics of symbiotic zooxanthellae in the coral Pocillopora damicornis exposed to elevated ammonium $\left(\mathrm{NH}_{4} \mathrm{Cl}\right)$ concentrations. Pac. Sci. 48, 263-272 (1994).

6. Muller-Parker, G., McCloskey, L. R., Hoegh-Guldberg, O. \& McAuley, P. J. Effect of ammonium enrichment on animal and algal biomass of the coral Pocillopora damicornis. Pac. Sci. 48, 273-283 (1994).

7. Smith, G. J. \& Muscatine, L. Cell cycle of symbiotic dinoflagellates: variation in $\mathrm{G}_{1}$ phase-duration with anemone nutritional status and macronutrient supply in the Aiptasia pulchella-Symbiodinium pulchrorum symbiosis. Mar. Biol. 134, 405-418 (1999).

8. Fitt, W. K. Cellular growth of host and symbiont in a cnidarian-zooxanthellar symbiosis. Biol. Bull. 198, 110-120 (2000).

9. Xiang, T. et al. Symbiont population control by host-symbiont metabolic interaction in Symbiodiniaceae-cnidarian associations. Nat. Commun. 11, 108 (2020).

10. Baghdasarian, G. \& Muscatine, L. Preferential expulsion of dividing algal cells as a mechanism for regulating algal-cnidarian symbiosis. Biol. Bull. 199, 278-286 (2000).

11. Dunn, S. R., Schnitzler, C. E. \& Weis, V. M. Apoptosis and autophagy as mechanisms of dinoflagellate symbiont release during cnidarian bleaching: every which way you lose. Proc. R. Soc. Lond. B 274, 3079-3085 (2007).

12. Pardee, A. B. $G_{1}$ events and regulation of cell proliferation. Science 246, 603-608 (1989).

13. Nishitani, H. \& Lygerou, Z. Control of DNA replication licensing in a cell cycle. Genes Cells 7, 523-534 (2002).

14. Stark, G. R. \& Taylor, W. R. Analyzing the $\mathrm{G}_{2} / \mathrm{M}$ checkpoint. Methods Mol. Biol. 280, 51-82 (2004).

15. Houtgraaf, J. H., Versmissen, J. \& van der Giessen, W. J. A concise review of DNA damage checkpoints and repair in mammalian cells. Cardiovasc. Revascularization Med. 7, 165-172 (2006).

16. Barnum, K. J. \& O’Connell, M. J. Cell-cycle regulation by checkpoints. Methods Mol. Biol. 1170, 29-40 (2014).

17. Malumbres, M. \& Barbacid, M. Cell cycle, CDKs and cancer: a changing paradigm. Nat. Rev. Cancer 9, 153-166 (2009).

18. Lim, S. \& Kaldis, P. CDKs, cyclins and CKIs: roles beyond cell-cycle regulation. Development 140, 3079-3093 (2013).

19. Vermeulen, K., Van Bockstaele, D. R. \& Berneman, Z. N. The cell cycle: a review of regulation, deregulation and therapeutic targets in cancer. Cell Prolif. 36, 131-149 (2003)

20. Cato, M. L. et al. Genome-wide analysis of cell-cycle-regulating genes in the symbiotic dinoflagellate Breviolum minutum. Genes Genomes Genet. 9, 3843-3853 (2019).

21. LaJeunesse, T. C. et al. Systematic revision of Symbiodiniaceae highlights the antiquity and diversity of coral endosymbionts. Curr. Biol. 28, 2570-2580 (2018).

22. Nitschke, M. R. et al. Description of Freudenthalidium gen. nov. and Halluxium gen. nov. to formally recognize clades Fr3 and H as Genera in the Family Symbiodiniaceae (Dinophyceae). J. Phycol. 56, 927-943 (2020).

23. Fujise, L. et al. Cell-cycle dynamics of cultured coral endosymbiotic microalgae (Symbiodinium) across different types (species) under alternate light and temperature conditions. J. Eukaryot. Microbiol. 65, 505-517 (2018).

24. Yuyama, I. \& Higuchi, T. Comparing the effects of symbiotic algae (Symbiodinium) clades C1 and D on early growth stages of Acropora tenuis. PLoS ONE 9, e98999 (2014).

25. Leal, M. C. et al. Symbiont type influences trophic plasticity of a model cnidarian-dinoflagellate symbiosis. J. Exp. Biol. 218, $858-863$ (2015).

26. Starzak, D. E., Quinnell, R. G., Nitschke, M. R. \& Davy, S. K. The influence of symbiont type on photosynthetic carbon flux in a model cnidarian-dinoflagellate symbiosis. Mar. Biol. 161, 711-724 (2014).

27. Gabay, Y., Weis, V. M. \& Davy, S. K. Symbiont identity influences patterns of symbiosis establishment, host growth, and asexual reproduction in a model cnidarian-dinoflagellate symbiosis. Biol. Bull. 234, 1-10 (2018).

28. Jeong, H. J. et al. Genetics and morphology characterize the dinoflagellate Symbiodinium voratum, n. sp., (Dinophyceae) as the sole representative of Symbiodinium clade E. J. Eukaryot. Microbiol. 61, 75-94 (2014).

29. Liu, H. et al. Symbiodinium genomes reveal adaptive evolution of functions related to coral-dinoflagellate symbiosis. Commun. Biol. 1, 1-11 (2018).

30. Wilkinson, S. P. aphid: an R package for analysis with profile hidden Markov models. Bioinformatics 35, 3829-3830 (2019)

31. Joubes, J. et al. CDK-related protein kinases in plants. Plant Mol. Biol. 43, 607-620 (2000).

32. Corellou, F., Camasses, A., Ligat, L., Peaucellier, G. \& Bouget, F.-Y. Atypical regulation of a green lineage-specific B-type cyclindependent kinase. Plant Physiol. 138, 1627-1636 (2005).

33. Malumbres, M. \& Barbacid, M. Mammalian cyclin-dependent kinases. Trends Biochem. Sci. 30, 630-641 (2005).

34. Talevich, E., Mirza, A. \& Kannan, N. Structural and evolutionary divergence of eukaryotic protein kinases in Apicomplexa. BMC Evol. Biol. 11, 321 (2011).

35. Darriba, D., Taboada, G. L., Doallo, R. \& Posada, D. ProtTest 3: fast selection of best-fit models of protein evolution. Bioinformatics 27, 1164-1165 (2011).

36. Stamatakis, A. RAxML version 8: a tool for phylogenetic analysis and post-analysis of large phylogenies. Bioinformatics 30, 13121313 (2014).

37. Cao, L. et al. Phylogenetic analysis of CDK and cyclin proteins in premetazoan lineages. BMC Evol. Biol. 14, 10 (2014).

38. Li, W., Jaroszewski, L. \& Godzik, A. Clustering of highly homologous sequences to reduce the size of large protein databases. Bioinformatics 17, 282-283 (2001).

39. Quevillon, E. et al. InterProScan: protein domains identifier. Nucleic Acids Res. 33, 116-120 (2005).

40. Chen, Y., González-Pech, R. A., Stephens, T. G., Bhattacharya, D. \& Chan, C. X. Evidence that inconsistent gene prediction can mislead analysis of dinoflagellate genomes. J. Phycol. 56, 6-10 (2020).

41. Guindon, S. \& Gascuel, O. A simple, fast, and accurate algorithm to estimate large phylogenies by maximum likelihood. Syst. Biol. 52, 696-704 (2003).

42. Anisimova, M. \& Gascuel, O. Approximate likelihood-ratio test for branches: a fast, accurate, and powerful alternative. Syst. Biol. 55, 539-552 (2006).

43. Guindon, S. et al. New algorithms and methods to estimate maximum-likelihood phylogenies: assessing the performance of PhyML 3.0. Syst. Biol. 59, 307-321 (2010).

44. Letunic, I. \& Bork, P. Interactive tree of life (iTOL) v4: recent updates and new developments. Nucleic Acids Res. 47, 256-259 (2019). 
45. Maor-Landaw, K., van Oppen, M. J. H. \& McFadden, G. I. Symbiotic lifestyle triggers drastic changes in the gene expression of the algal endosymbiont Breviolum minutum (Symbiodiniaceae). Ecol. Evol. 10, 451-466 (2020).

46. Shoguchi, E. et al. Draft assembly of the Symbiodinium minutum nuclear genome reveals dinoflagellate gene structure. Curr. Biol. 23, 1399-1408 (2013).

47. Dobin, A. et al. STAR: ultrafast universal RNA-seq aligner. Bioinformatics 29, 15-21 (2013).

48. Liao, Y., Smyth, G. K. \& Shi, W. featureCounts: an efficient general purpose program for assigning sequence reads to genomic features. Bioinformatics 30, 923-930 (2014).

49. Robinson, M. D., McCarthy, D. J. \& Smyth, G. K. edgeR: a Bioconductor package for differential expression analysis of digital gene expression data. Bioinformatics 26, 139-140 (2010).

50. Team, R. C. R: A Language and Environment for Statistical Computing. Vienna: R Project. Available at: https://www.R-project.org (2020).

51. Law, C. W., Chen, Y., Shi, W. \& Smyth, G. K. voom: precision weights unlock linear model analysis tools for RNA-seq read counts. Genome Biol. 15, R29 (2014).

52. Santamaría, D. et al. CDK1 is sufficient to drive the mammalian cell cycle. Nature 448, 811 (2007).

53. Lee, M. G. \& Nurse, P. Complementation used to clone a human homologue of the fission yeast cell-cycle control gene cdc2. Nature 327, 31 (1987).

54. Mendenhall, M. D. \& Hodge, A. E. Regulation of Cdc28 cyclin-dependent protein kinase activity during the cell cycle of the yeast Saccharomyces cerevisiae. Microbiol. Mol. Biol. Rev. 62, 1191-1243 (1998).

55. Huang, D., Friesen, H. \& Andrews, B. Pho85, a multifunctional cyclin-dependent protein kinase in budding yeast. Mol. Microbiol. 66, 303-314 (2007).

56. Carroll, A. S. \& O'Shea, E. K. Pho85 and signaling environmental conditions. Trends Biochem. Sci. 27, 87-93 (2002).

57. Li, S. et al. The cdc2-related kinase, PISSLRE, is essential for cell growth and acts in $\mathrm{G}_{2}$ phase of the cell cycle. Cancer Res. 55, 3992-3995 (1995).

58. Malumbres, M. Cyclin-dependent kinases. Genome Biol. 15, 122 (2014).

59. Ma, Z. et al. Phylogenetic analysis reveals the evolution and diversification of cyclins in eukaryotes. Mol. Phylogenet. Evol. 66, 1002-1010 (2013).

60. Schachter, M. M. et al. A CDK7-CDK4 T-loop phosphorylation cascade promotes $\mathrm{G}_{1}$ progression. Mol. Cell 50, 250-260 (2013).

61. Larochelle, S. et al. Requirements for CDK7 in the assembly of CDK1/cyclin B and activation of CDK2 revealed by chemical genetics in human cells. Mol. Cell 25, 839-850 (2007).

62. Jones, N. G. et al. Regulators of Trypanosoma brucei cell-cycle progression and differentiation identified using a kinome-wide RNAi screen. PLoS Pathog. 10, e1003886 (2014).

63. Tu, X. \& Wang, C. C. Pairwise knockdowns of cdc2-related kinases (CRKs) in Trypanosoma brucei identified the CRKs for $\mathrm{G}_{1} / \mathrm{S}$ and $\mathrm{G}_{2} / \mathrm{M}$ transitions and demonstrated distinctive cytokinetic regulations between two developmental stages of the organism. Eukaryot. Cell 4, 755-764 (2005).

64. Ortega, S., Malumbres, M. \& Barbacid, M. Cyclin D-dependent kinases, INK4 inhibitors and cancer. Biochim. Biophys. Acta 1602, 73-87 (2002).

65. Roques, M. et al. Plasmodium P-type cyclin CYC3 modulates endomitotic growth during oocyst development in mosquitoes. PLoS Pathog. 11, e1005273 (2015).

66. Peng, L., Skylar, A., Chang, P. L., Bisova, K. \& Wu, X. CYCP2;1 integrates genetic and nutritional information to promote meristem cell division in Arabidopsis. Dev. Biol. 393, 160-170 (2014).

67. Huang, D. et al. Cyclin partners determine Pho85 protein kinase substrate specificity in vitro and in vivo: control of glycogen biosynthesis by Pcl8 and Pcl10. Mol. Cell. Biol. 18, 3289-3299 (1998).

68. Barott, K. L., Venn, A. A., Perez, S. O., Tambutté, S. \& Tresguerres, M. Coral host cells acidify symbiotic algal microenvironment to promote photosynthesis. Proc. Natl. Acad. Sci. 112, 607-612 (2015).

69. Alvarez, C. A. \& Suvorova, E. S. Checkpoints of apicomplexan cell division identified in Toxoplasma gondii. PLoS Pathog. 13, e1006483 (2017).

70. Measday, V. et al. A family of cyclin-like proteins that interact with the Pho85 cyclin-dependent kinase. Mol. Cell. Biol. 17, 1212-1223 (1997).

71. Jiang, M., Gao, Y., Yang, T., Zhu, X. \& Chen, J. Cyclin Y, a novel membrane-associated cyclin, interacts with PFTK1. FEBS Lett. 583, 2171-2178 (2009).

72. Sun, T., Co, N. N. \& Wong, N. PFTK1 interacts with cyclin Y to activate non-canonical Wnt signaling in hepatocellular carcinoma. Biochem. Biophys. Res. Commun. 449, 163-168 (2014).

73. MacDonald, B. T., Tamai, K. \& He, X. Wnt/ $\beta$-catenin signaling: components, mechanisms, and diseases. Dev. Cell 17, 9-26 (2009).

74. Leander, B. S. \& Keeling, P. J. Early evolutionary history of Dinoflagellates and Apicomplexans (Alveolata) as inferred from Hsp90 and actin phylogenies. J. Phycol. 40, 341-350 (2004).

75. Liu, Y., Hu, H. \& Li, Z. The cooperative roles of Pho80-like cyclins in regulating the $\mathrm{G}_{1} / \mathrm{S}$ transition and posterior cytoskeletal morphogenesis in Trypanosoma brucei. Mol. Microbiol. 90, 130-146 (2013).

76. Stat, M. \& Gates, R. D. Clade D Symbiodinium in scleractinian corals: a "nugget" of hope, a selfish opportunist, an ominous sign, or all of the above?. J. Mar. Biol. 2011, 1-10 (2010).

77. Keeling, P. J. et al. The Marine Microbial Eukaryote Transcriptome Sequencing Project (MMETSP): illuminating the functional diversity of eukaryotic life in the oceans through transcriptome sequencing. PLoS Biol. 12, e1001889 (2014).

78. Duncan, R. P., Feng, H., Nguyen, D. M. \& Wilson, A. C. C. Gene family expansions in aphids maintained by endosymbiotic and nonsymbiotic traits. Genome Biol. Evol. 8, 753-764 (2016).

79. Chen, Y., González-Pech, R. A., Stephens, T. G., Bhattacharya, D. \& Chan, C. X. Evidence that inconsistent gene prediction can mislead analysis of dinoflagellate genomes. J. Phycol. 1, 95 (2019).

\section{Acknowledgements}

This research was supported by the Marsden Fund of the Royal Society Te Apārangi of New Zealand (Grant Number VUW1601), awarded to S.K.D., A.R.G., V.M.W. and C.A.O. and an associated PhD scholarship to L.M.G. Additional support was provided via strategic research funds from the School of Biological Sciences at Victoria University of Wellington to S.K.D.

\section{Author contributions}

The study was conceptualised by L.M.G., C.A.O., A.R.G., V.M.W., and S.K.D. L.M.G. wrote the manuscript, collected and analysed the sequence and phylogenetic data, and constructed all figures (apart from figure 3) and all tables. S.P.W. helped L.M.G. with data collection and analysis. S.A.K. conducted and analysed the expression analysis, constructed figure 3 , helped revise the manuscript, and advised on methods, data analysis and 
visualisation, C.A.O., V.M.W., S.K.D., A.R.G. helped edit and refine the manuscript. All authors reviewed the manuscript before submission.

\section{Competing interests}

The authors declare no competing interests.

\section{Additional information}

Supplementary information is available for this paper at https://doi.org/10.1038/s41598-020-76621-1.

Correspondence and requests for materials should be addressed to S.K.D.

Reprints and permissions information is available at www.nature.com/reprints.

Publisher's note Springer Nature remains neutral with regard to jurisdictional claims in published maps and institutional affiliations.

(c) (i) Open Access This article is licensed under a Creative Commons Attribution 4.0 International License, which permits use, sharing, adaptation, distribution and reproduction in any medium or format, as long as you give appropriate credit to the original author(s) and the source, provide a link to the Creative Commons licence, and indicate if changes were made. The images or other third party material in this article are included in the article's Creative Commons licence, unless indicated otherwise in a credit line to the material. If material is not included in the article's Creative Commons licence and your intended use is not permitted by statutory regulation or exceeds the permitted use, you will need to obtain permission directly from the copyright holder. To view a copy of this licence, visit http://creativecommons.org/licenses/by/4.0/.

(c) The Author(s) 2020 